\title{
Endovesical Metastasis of a Colic Adenocarcinoma: A Case Report
}

\author{
Mohamed Bouziane ${ }^{1, ~ *, ~ O m a r ~ M o u n i ~}{ }^{1}$, Wafaa Kaikani ${ }^{2}$, Meriem Kassimi ${ }^{3}$, Redouane Rabii ${ }^{4}$, \\ Abderrahmane Albouzidi ${ }^{5}$ \\ ${ }^{1}$ Department of General Surgery, Cheikh Khalifa Ibn Zaid International University Hospital, Mohammed VI University of Health Sciences \\ (UM6SS), Casablanca, Morocco \\ ${ }^{2}$ Department of Oncology, Cheikh Khalifa Ibn Zaid International University Hospital, Mohammed VI University of Health Sciences \\ (UM6SS), Casablanca, Morocco \\ ${ }^{3}$ Department of Radiology, Cheikh Khalifa Ibn Zaid International University Hospital, Mohammed VI University of Health Sciences \\ (UM6SS), Casablanca, Morocco \\ ${ }^{4}$ Department of Urology, Cheikh Khalifa Ibn Zaid International University Hospital, Mohammed VI University of Health Sciences (UM6SS), \\ Casablanca, Morocco \\ ${ }^{5}$ Department of Anatomopathology, Cheikh Khalifa Ibn Zaid International University Hospital, Mohammed VI University of Health Sciences \\ (UM6SS), Casablanca, Morocco
}

\section{Email address:}

mbouziane@um6ss.ma (M. Bouziane), mouniomar@gmail.com (O. Mouni), wafaakaikani@yahoo.fr (W. Kaikani), meriem.kassimi@live.fr (M. Kassimi), rabiibest@yahoo.fr (R. Rabii), albouzidi@gmail.com (A. Albouzidi)

${ }^{*}$ Corresponding author

\section{To cite this article:}

Mohamed Bouziane, Omar Mouni, Wafaa Kaikani, Meriem Kassimi, Redouane Rabii, Abderrahmane Albouzidi. Endovesical Metastasis of a Colic Adenocarcinoma: A Case Report. Journal of Surgery. Vol. 9, No. 1, 2021, pp. 45-48. doi: 10.11648/j.js.20210901.18

Received: January 29, 2021; Accepted: February 9, 2021; Published: February 27, 2021

\begin{abstract}
Background: Bladder infiltration by neighbourhood tumors is a common situation and can be found in prostate, colorectum and genital tumours in women. The main mechanism is a direct invasion of the bladder by the tumor. Intraluminal bladder metastases from distant primary tumors remain exceptional. Patient: We report the case of a 56-year-old patient without any particular medical history initially admitted for an occlusion caused by a transverse colic tumor. The patient did not have clinically haematuria and the initial scan did not show a location or abnormality in the bladder. Initial surgical exploration did not find peritoneal or bladder abnormalities. The patient's evolution will be marked by the appearance of bladder thickening at a distance from the primary tumor and peritoneal carcinosis. The immunohistochemical study in addition to the anatomopathological examination resulted in positive marking of the chorion tubes by anti CDX2 and anti CK 20 and positive marking of surface vesical epithelium by anti P63, anti GATTA3 and anti CK7 which confirmed the colorectal origin of endo-vesical metastasis. Conclusion: We will try through this case report and a review of literature to shed light on this unusual situation and it is important to keep in mind the possibility of remote metastatic location at the bladder of another primary tumor.
\end{abstract}

Keywords: Adenocarcinoma, Colic, Metastasis, Bladder

\section{Introduction}

Colorectal cancer is a common tumor whose usual metastatic sites are mainly the liver, lung, bone, brain and peritoneum. [1]

Adenocarcinoma remains an unusual form of bladder cancer and accounts for only 0.5 to $2 \%$ of the total of bladder tumours. [2, 3]
Most often, this adenocarcinoma is the result of the extension of adenocarcinomas developing at the level of the neighbouring organs such as prostate tumours, colo-rectum and genital tumors in women.

\section{Case Report}

We report a case of a 56-year-old patient, 10-pack-year 
smoker, with no other medical history, never operated on and who is admitted to the emergency department for occlusive syndrome on stenosis tumor of the left transverse colon made of abdominal pain, no gas passing with vomiting evolving for 4 days without rectal bleeding and apyrexia.

The examination of the abdomen reveals abdominal distension, tympanic sound without tenderness, no obvious hernia. rectal touch did not find abnormalities.

The abdominal scan revealed a colic occlusion on left colic angle tumour without visualizing liver metastases, peritoneal carcinosis or bladder location.

An exploratory laparotomy found an occlusion on a left transverse colon tumor in a completely stenosing virole with the presence at $2 \mathrm{~cm}$ of the primary tumour of a suspect peritoneal nodule. (Figure 1)

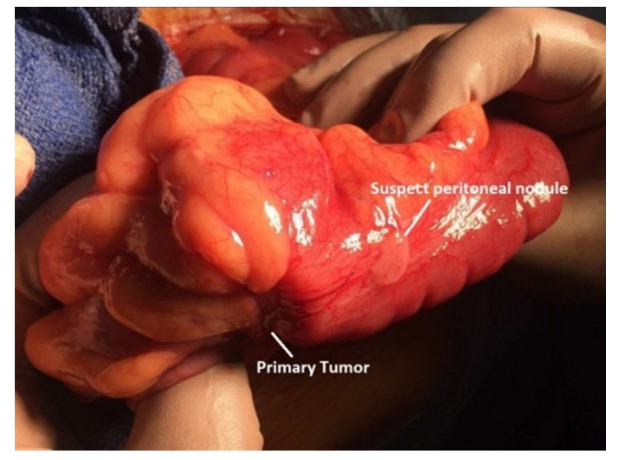

Figure 1. Aspect of the primary tumour and a suspect peritoneal nodule.

This exploration did not reveal liver metastases or other colic, peritoneal or bladder location.

A discharge colostomy with biopsy of the peritoneal nodule and biopsy of the tumor through the stoma were performed.

The result of the anatomopathology of the tumor and the peritoneal nodule was in favour of a moderately differentiated and invasive adenocarcinoma.

The extension check-up was normal, colonoscopy did not find any other colic locations, the carcinoembryonic antigen was normal at $0.65 \mathrm{ng} / \mathrm{ml}$ and the decision of the multidisciplinary consultation meeting was to perform tumor surgery.

Per operative exploration found multiple nodules of diffuse peritoneal carcinosis with advanced local invasion and intimate adhesion of a proximal small intestine handle to the tumor without liver metastases or other locations.

An abstention from surgery is decided with the completion of multiple biopsies whose anatomopathological examination revealed a diffuse peritoneal location of colic adenocarcinoma.

The patient received 6 cures of FOLFOX-based chemotherapy.

The Thoraco-abdomino-pelvic control scanner noted a partial regression of the left transverse colon tumour and the appearance of an apical bladder thickening of $15 \mathrm{~mm}$ without any remote metastasis, especially not in the liver or lung. (Figure 2)
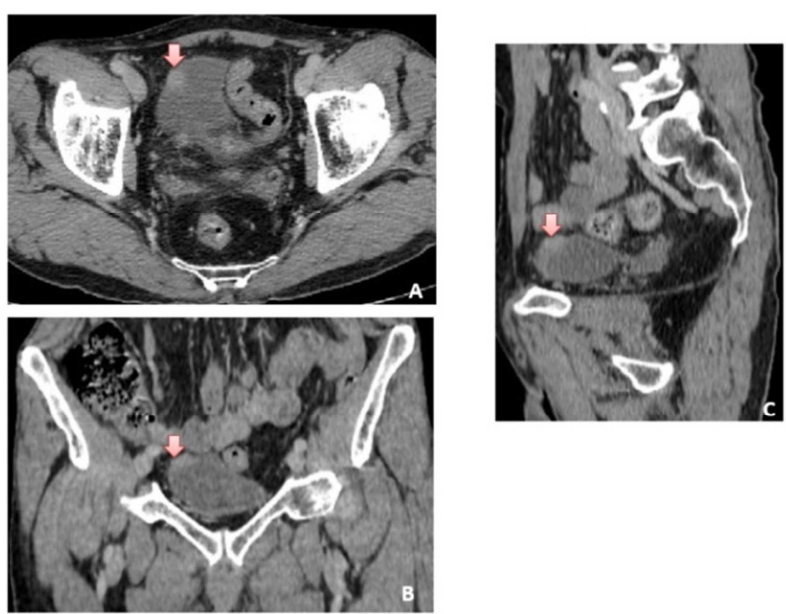

Figure 2. Scannographique images after contrast injection into the axial (A), Coronal (B) and Sagittal (C) planes: an irregular budding thickness of the right anterolateral wall of the bladder.

The patient did not have haematuria or other associated urinary signs.

A cystoscopy revealed a bladder dome tumor and an endoscopic resection was performed.

The anatomopathological and immunohistochemical study resulted in positive marking of the chorion tubes by anti CDX2 and anti CK 20 and positive marking of surface vesical epithelium by anti P63, anti GATTA3 and anti CK7 which is in favour of a vesical endo extension of a colic adenocarcinoma. (Figures 3 and 4)

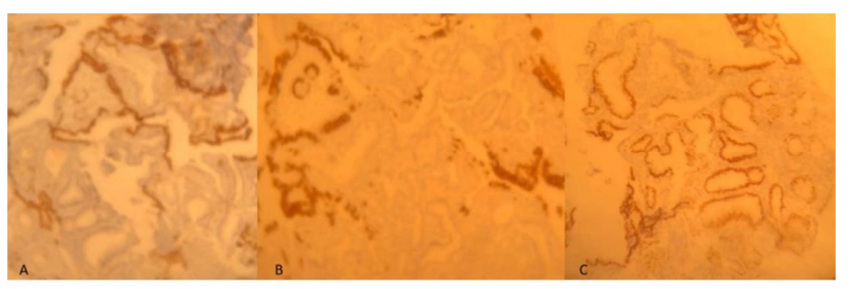

Figure 3. Magnification $x 100$.

A: Tumor cells do not express P63 while expressed by surface urothelium B: Tumor cells do not express cytokeratin 7 while expressed by urothelium $\mathrm{C}$ : tumor cells express CDX2 not expressed by urothelium

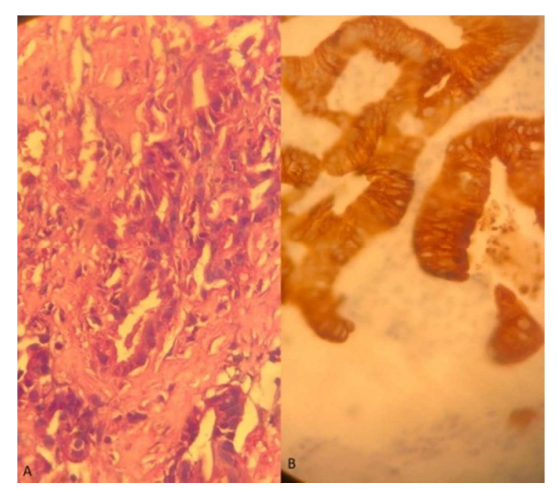

Figure 4. Magnification $x 400$.

A: Malignant tumor proliferation made of anastomotic tubes B: immunohistochemical study auto Stainer Dako: tumor cells express cytokeratin 20 


\section{Discussion}

Bladder adenocarcinoma accounts for only 0.5 to $2 \%$ of the total primary bladder tumors. [2]

Primary and metastatic adenocarcinomas of urinary bladder are morphologically similar, but histogenetically different. [3]

Intraluminal bladder metastases from distant primary tumors remain extremely rare.

Colorectal adenocarcinoma is the most frequent metastasis in the bladder. It is important to differentiate primary bladder adenocarcinoma from secondary colorectal adenocarcinoma.

However, it is generally difficult based on morphologic features, especially on small biopsy specimens, as they share similar histologic features. [4]

Previous renal pelvis and ureteral cancers, particularly when diagnosed at an early age, increase the risk for subsequent CRC. Likewise, a history of CRC, especially in cases with multiple primary tumors, is associated with an increased risk of renal pelvis and ureteral cancers. These findings support a possible common pathogenetic mechanism between CRC and urologic cancers. [5]

Klinger's study of 5,000 autopsies at Henry Ford Hospital on secondary tumors of the genitourinary tract revealed the following data: only $0.66 \%(33 / 5000)$ of cases were adenocarcinomas. [6]

Only $0.06 \%$ (3/5000) were of colorectal origin.

Ganem, Batal et al. Have on a review of literature found 80 cases of metastasis at the bladder level. The origin of these metastases was the stomach ( 25 cases), melanomas (18 cases), breast (16 cases and lungs ( 3 cases).

Only 4 cases of recto-colic cancers were implicated, including 1 in caecum and 3 recto-sigmoid. [7]

The mechanism of these distant metastases remains unknown and would require tumor cells to be able to pass through the vessels, create a vascular embolus that must survive in the bloodstream, stop and then externalize the capillaries and multiply at the level of the organ concerned [8].

Haematuria is the most common sign of revelation of primitive bladder carcinomas.

The presence of urinary signs such as haematuria reflecting bladder irritation appears to depend on the ability of tumour embolisms to grow rapidly, penetrate the bladder epithelium and reach bladder light prior to the patient's death from the aggressiveness of the primary tumour [9].

The occurrence of haematuria in metastatic localization is rare due to the rarity of the occurrence of ulceration of the vesical mucosa.

Hermann reported these histopathological findings: the mucous membrane is generally spared in gastrointestinal vesical metastases [10].

Sheehan and Greenberg reported similar findings: only 3 of 21 patients with bladder metastases had macroscopic haematuria [11].

In our patient, the bleeding appeared late and required hospitalization with transfusion.

Immunohistochemistry improved the diagnostic sensitivity of the anatomopathological examination [12].

The expression of cytokeratin 20 appears to be limited to gastrointestinal epithelial cells [13-15].

$95 \%$ of colorectal cancer cases can be detected by this immunohistochemical examination. [16]

Our anatomopathological examination suspected colorectal metastasis at the bladder and the diagnosis was confirmed by immunohistochemical examination.

\section{Conclusion}

In summary, the colon is a very rare cause of remote bladder metastasis.

Patients with a primary diagnosis of CRC, especially those of a younger age, should be considered at increased risk for specific urologic cancers.

Although this situation is rare, it is always important to keep in mind the possibility of remote metastatic location at the bladder, especially in the presence of another synchronous primary tumor.

\section{References}

[1] Morson BC, Dawson IMP. Gastrointestinal Pathology. 4th ed, Malden Oxford: Blackwell Science 2003; 1532.

[2] Tuna B. Adenocarcinoma of the Urinary Bladder. J Urol Surg 2018; 5 (4): 233-238.

[3] Kumari N, Vasudeva P, Kumar A, Agrawal U. Adenocarcinoma of urinary bladder: A report of two patients. J Can Res Ther 2015; 11: 1033.

[4] Dadhania V, Czerniak B, Guo CC. Adenocarcinoma of the urinary bladder. Am J Clin Exp Urol 2015; 51-63.

[5] Calderwood AH, Huo D, Rubin DT. Association Between Colorectal Cancer and Urologic Cancers. Arch Intern Med. 2008; 168 (9): 1003-1009. doi: 10.1001/archinte.168.9.1003.

[6] Klinger ME. Secondary tumors of the genitourinary tract. $J$ Urol 1951; 65: 144-53.

[7] Ganem EJ, Batal JT. Secondary malignant tumors of the urinary bladder metastatic from primary foci in distant organs. J Urol 1956; 75: 965-72.

[8] Gutman M, Fidler IJ. Biology of human colon cancer metastases. World J Surg 1995; 19: 226-34.

[9] Daroca PJ, Mackenzie F, Reed RJ, Keane JM. Primary adenovillous carcinoma of the bladder. J Urol 1976; 115: 41-5.

[10] Hermann HB. Metastatic tumors of the urinary bladder originating from the carcinoma of the gastrointestinal tract. $J$ Urol 1929; 22: 257-73.

[11] Sheehan EE, Greenberg SD, Scott R. JR. Metastatic neoplasms of the bladder. J Urol 1963; 90: 281-4.

[12] Merrie AEH, van Rij AM, Dennelt ER, Phillips LV, Yun K, McCall JL. Prognostic significance of occult metastases in colon cancer. Dis Colon Rectum 2003; 46: 221-31. 
[13] Wauters CC, Smedts F, Gerrits LG, Bosman FT, Ramaekers FC. Keratins 7 and 20 as diagnostic markers of carcinomas metastatic to the ovary. Hum Pathol 1995; 26: 852-5.

[14] Denis MG, Lipart C, Leborgne J, et al. Detection of disseminated tumor cells in peripheral blood of colorectal cancer patients. Int J Cancer 1997; 74: 540-4.
[15] Litle VR, Warren RS, Moore DN, Pallavicini MG. Molecular cytogenetic analysis of cytokeratin 20 -labeled cells in primary tumors and bone marrow aspirates from colorectal carcinoma patients. Cancer 1997; 79: 1664-70.

[16] Moll R, Lowe A, Laufer J, FrankeWW. Cytokeratin 20 in human carcinomas. A new histodiagnostic marker detected. 